\title{
On The Subtitle Translation of Mirror Mirror from Relevance Theory
}

\author{
Han Cui-ping ${ }^{1}$, Ni Xue-li \\ Yangtze University, College of Arts and Science (Jingzhou. Hubei, 434020)
}

\begin{abstract}
The popularity of foreign audio-visual products facilitates exchanges and communication among various cultures, and recent years have seen an increasing number of films distributed in foreign markets, which naturally leads to the prosperity of subtitle translation. This thesis selects the film Mirror Mirror as a case to analyze film subtitle translation strategies in the perspective of relevance theory. It consists of five parts: Part One offers a brief introduction to the research background and issues together with thesis structure. Partr Two offers some theories of Relevance Theory and relationship between relevance theory and subtitle translation. Part Three focuses a study on the subtitle translation strategies of Mirror Mirror from the perspective of relevance theory in which a detailed analysis of the difficult problems and response strategies will be made. Part Four is the conclusion. The thesis is an attempt to prove that Relevance Theory can be well applied in subtitle translation to guide the translation activity and improve the quality of subtitle translation.
\end{abstract}

Keywords: Subtitle translation; Relevance Theory; Mirror Mirror; translation strategies.

\section{INTRODUCTION}

Throughout the translation history, equivalence has been regarded as the vital standard for a very long time. Nevertheless, with translation fields have become more and more colorful and diversity, such as film subtitle translation, business translation and advertising translation, the limitations of these translations rules have gradually turned up. Thus, new theories are very in need. Especially in the film subtitle translation. In early days all the films have no sound and there were no such problem about movies dubbing or film subtitle translation. And with the development of sound films. These problems of we should take which translation methods to translate film subtitle have got a lot of attentions from film circle and related entertainment world. How to make audience understand films easier. So it is obvious that film subtitle translation is of great significance to us.

In addition, the relevance translation theory turned up to us and caught wide attentions as it is a powerful pragmatics and revolutionary theory. Relevance theory believes that relevance is a property which need not be represented, let alone, or computed, in order to be realized. When it is represented, it is represented in terms of comparative judgments and gross absolute judgments, but not in terms of fine absolute judgments, i.e. the quantitative ones (Sperber and Wilson, 1986/1995: 132). So, relevance theory gives the following extent conditions of relevance: Extent condition 1: An assumption is relevant in context to the extent that its contextual effects in this context are large. Extent condition 2: An assumption is relevant in a context to the extent that the effort required to process it in this context is small Relevance theory believes that in the process of communication, the addresser and the addressee need not consider the problem of co-operation because communication depends on relevance. Sperber and Wilson have proposed two principle of relevance, the first termed as communicative principle and the second termed as cognitive principle. The latter are, however, not the principle communicators abide by, but the general principle used to explain cognitive procedure in communication. Every utterance (or other act of inferential communication) communicates a presumption of its own optimal relevance. Or Human cognition tends to be geared to the maximization of relevance.

This thesis takes the film-Mirror Mirror as an example to make a case study about relevance theory applied in film subtitle translation.

${ }^{1}$ Corresponding Author: candyhan2006@163.com. 
The thesis consists of four parts. Chapter one offers a brief introduction. Part Two offers some theories of Relevance Theory and relationship between relevance theory and subtitle translation. Part Three focuses a study on the subtitle translation strategies of Mirror Mirror from the perspective of relevance theory in which a detailed analysis of the difficult problems and response strategies will be made. Part Four is the conclusion.

\section{RELEVANCE THEORY AND SUBTITLE TRANSLATION}

\subsection{Relevance Theory}

The key concepts of relevance theory are "communication" and"cognition". The central claim of relevance theory is that the expectations of relevance raised by an utterance are precise enough, and predictable enough, to guide the hearer towards the speaker's meaning. The aim is to explain in cognitively realistic terms what these expectations of relevance amount to, and how they might contribute to an empirically plausible account of comprehension. Here, I will introduce some important parts of this theory.

Relevance theory believes that relevance is a property which need not be represented, let alone, or computed, in order to be realized. When it is represented, it is represented in terms of comparative judgments and gross absolute judgments, but not in terms of fine absolute judgments, i.e. the quantitative ones (Sperber and Wilson, 1986/1995: 132). So, relevance theory gives the following extent conditions of relevance: Extent condition 1: An assumption is relevant in context to the extent that its contextual effects in this context are large. Extent condition 2: An assumption is relevant in a context to the extent that the effort required to process it in this context is small Relevance theory believes that in the process of communication, the addresser and the addressee need not consider the problem of co-operation because communication depends on relevance. Sperber and Wilson have proposed two principle of relevance, the first termed as communicative principle and the second termed as cognitive principle. The latter are, however, not the principle communicators abide by, but the general principle used to explain cognitive procedure in communication. Every utterance (or other act of inferential communication) communicates a presumption of its own optimal relevance. Or (Every ostensive stimulus conveys a presumption of its own optimal relevance) Human cognition tends to be geared to the maximization of relevance.

According to relevance theory, humans automatically aim at maximal relevance, i.e. maximal cognitive effect for minimal processing effort. It is regarded as the most general factor that determines the course of human information processing and determines which information is paid attention to, which background assumptions are retrieved from memory and used as context, and which inferences are drawn.

Relevance Theory claims that in every case, the task of the addressee is to find an interpretation consistent with the principle of relevance that is an interpretation which the communicator could manifestly have expected to be optimally relevant. Hence, Sperber and Wilson propose the presumption of optimal relevance.

Presumption of optimal relevance:

- The ostensive stimulus is relevant enough for it to be worth the addressee's effort to process it.

- The ostensive stimulus is the most relevant one compatible with the communicator's abilities and preferences. (Sperber \&Wilson, 1995:270)

According to clause (a) of the presumption of optimal relevance, the audience is entitled to expect the ostensive stimulus to be at least relevant enough to be worth processing.

According to clause (b) of the presumption of optimal relevance, the audience of an ostensive stimulus is entitled to even higher expectations than this. The communicator wants to be understood. It is therefore in her interest—within the limits of her own capabilities and preferences - to make her ostensive stimulus as easy as possible for the audience to understand, and to provide evidence not just for the cognitive effects she aims to achieve in her audience but also for further cognitive effects which, by holding his attention, will help her achieve her goal.

When processing ostensive stimulus made by the speaker, the hearer hopes to have the optimal relevance. If an utterance has adequate contextual affects, and at the same time, the hearer needs only to make the smallest effort to 
understand that, the utterance has the optimal relevance. To make the stimulus have the optimal relevance, two conditions should be satisfied: the contextual effects of an utterance are enough to attract the hearer's attention, the hearer has made efforts to acquire the contextual effects. In other words, to have the optimal relevance, the speaker not only should supply the hearer appropriate contextual effects, but also should try to make the hearer obtain the contextual effects with the smallest efforts.

\subsection{Relevance Theory and Film Subtitle Translation}

Gutt (1991), a cognitive pragmatist, proposes that Relevance Theory is an explicit general theory of translation, which has important significance for the translation. In fact, what Gutt intends to do is build up a new translation theory by Relevance Theory; however, to his surprise, he finds that Relevance Theory itself has strong explanation for translation.

As one type of translation, subtitles translation can also be explained by Relevance Theory, which has strong explanation for subtitles translation. Relevance Theory, as an explicit general theory of translation, is also suitable for subtitles translation. Relevance Theory states that human employs inference to communicate, audience is supposed to infer from the stimuli of the speaker's intended information. Translation is under the interpretive use of language. It means that translation is required to rearrange the source language using the target language. As a matter of fact, subtitles translation refers to use one language to express another language and try the best to make it resemble to the original utterance, making the target language audience understand the original as quickly as possible. It proves that subtitles translation can also be considered as interlingual interpretive use.

Subtitles translation is also a cross-cultural communicative activity, that is, it is a two-stage communicative activity. One is the communication between the original author and the translator, and the other refers to the translator and the target viewer. Both the two communications are guided by Relevance Theory. The subtitler should convey the idea which is the most relevant to explain the original text. Therefore, the target language audience can reproduce the ideas quickly based on relevance principle. Subtitles translation, as a form of the communication, should ensure the success of the communication. Subtitles translation makes intentions and expectations meet. In order to make the communication successful, what the subtitler must do is to make his intentions meet the audience's expectations.

The process of movie subtitle translation is not merely a conversion between two languages, but a process of transmission and transplant in which cultural exchange is included. The translator is, therefore, required not only to interpret the original intention of the source text as completely as possible, but the cultural information as well. That is to say, it should be assured that the effort of acquiring information from the audience should be minimized yet the understanding should be maximized, meanwhile the result of information transmission should be noticed to achieve the optimal relevance. Here, only three contextual factors are concerned during the translator's transmitting and understanding process of the original intention, i.e. contextual relevance, cultural relevance and recipient relevance.

\subsubsection{Contextual Relevance}

There is a certain context contained in every single movie, it doesn't refer to any specific situational context but an even larger social and cultural background. The context of subtitle translation usually crosses two different situational contexts and social cultural backgrounds ---the context of the movie and the context of the translated subtitle which are strongly and internally related. Therefore, while translating subtitle, the translator should not only take full consideration of all sorts of specific factors, such as emotional factor, social factor, historical, cultural and background factors, but the relevance between these factors and the target language's context as well. Hence, the translator should consider the relevance as the foundation of translation process. The only way of realizing the recipients' comprehension to the story and therefore appreciating the movie plot is to emphasize the transmission of the implied communicative purpose in the language during the ostensive-inferential process.

\subsubsection{Cultural Relevance}

Language is a part of culture, in the mechanism of interlingual transition, the cultural factor is playing a very important role due to similar cultural forms share similar information channels; conversely, differences will definitely be generated under various forms of culture and therefore block the information channel. 
English movies contain a lot of language which has specific cultural significances bearing specific cultural information, like values, ways of thinking, religions, manners and customs, etc. These cultural differences may cause the recipient's difficulty in accepting and understanding. During the translation of subtitle, the translator must not only provide semantic information in the dialogue, but also adopt different strategies in coping with the cultural image and making the implication understandable by the recipient. That is to say, to make the recipient uses the minimum effort in understanding maximum cultural information. The incapability of delivering the cultural image would cause the confusion of the recipient and make whom hard to understand the connotation of the movie. So, it's the translator's obligation to deliver the cultural information contained in the movie to the recipient as much as possible and thus achieving the prosperity of communication between different cultures.

\subsubsection{Recipient Relevance}

There is a tendency or an old view in traditional translation theory that the translator only needs to take responsibility on the source text. However, translation is not a closed, self-reliant or one-way decided activity; it has been commercialized and marketized especially for movie subtitle. Only when the recipient as a customer satisfied with the translated subtitle can the value and recognition of the translator be realized.

During the subtitle translation, first, due to the limitation of space and time, the length of one piece of subtitle should meet with the desire of average audience. Only in this way can the recipient possess an appropriate pace while viewing; second, the translator should take the following factors that may affect a recipient into consideration during the translation process: the receptiveness, initiative, variability and social class diversity. Try not to use too many unfamiliar words or sinicized idioms or sayings and cautiously treat the foreign allusions that are hard to be understood by Chinese recipients. All in all, the ultimate goal of a movie subtitle translator is to put the recipient's benefits and understanding effect as the crucial point and to make the recipient capable of understanding the whole movie with minimum effort.

\section{A Study On the Subtitle Translation Strategies of Mirror Mirror from Relevance THEORY}

\subsection{Brief Introduction of Mirror Mirror}

Mirror Mirror is a 2012 Czech-American family adventure comedy-drama fantasy film based on the fairy tale "Snow White" collected by the Brothers Grimm. The film received an Academy Award nomination for Best Costume Design . With the effective subtitle translation, it is also well received by Chinese audience and made a leading box-office record of more than 30 million Dollars in the first week on show.

The main plot of the film is both conventional and rebellious. In order to despoil political power, the vicious queen wields the black magic to turn the king into man-eating monster. What's worse, since the queen is the nature of waste, country was sunk into a severe economic crisis, people have to displace and emigrate. To avenge her father and take people from this nightmare, Snow white defeat the queen successfully and recapture the regime with the help of seven dwarves.

\subsection{Analysing the Mirror Mirror's subtitle translation}

\subsubsection{Omission}

Film subtitles, as a special type of texts, have their own features as different from other genres of texts such as literary ones. As a constrained form of translation, i.e. it's subject to spatial and temporal limitations; subtitle translation distinguishes itself from other forms of translation by frequently employing the strategy of omission to meet translation challenges. This is accountable by Sperber and Wilsion's theory of "optimal relevance". There are mainly two restrictions for the translation of the subtitles, space and time restrictions. Translators must try to ensure the minimum of the space of subtitles, and offer adequate reading time for the audience to obtain the necessary information for understanding of the plot. Because of the spatial and temporal constraints, the subtitle has to convey essential messages within limited time and space while achieving a "optimal relevance". In the meantime, film viewers do not receive information just through the subtitles, as the pictures, sounds, actions etc. are all important 
channels through which the viewers get reductive translation in subtitling, whereby the translation ensures "the least effort" of the viewers, thus achieving "optimal relevance" .Due to the time and space constraints of subtitles, the movie subtitles require to be concise and easy to understand. Omission refers to omit certain information which is unnecessary and keep the vital information. It is used when the information will misunderstand the target language viewers. In addition, it can make the lines concise and save some space, which is used for the target language audience to best understand the movie by omitting many distracting and unnecessary words or sentences instead of obtaining the wrong contextual effects.

Sometimes, due to the limitation of time and space, some information that is of minor importance needn't be translated. Like messages already delivered through the picture, or meaningless articles and so on. Omission is another reflection on Relevance Theory that some information needs to be deduced by the viewer to achieve relevance; when such information can be inferred under the context, there is no need to translate so much details. E.g.

S1a.Once upon a time, in a kingdom, far far away .

$\mathrm{S} 1 \mathrm{~b}$ 很久以前, 在一个遥远的国家里。

S2a Perhaps you need to see for yourself what goes on in your kingdom.

S2b 也许您需要视察一下您的王国了。

S3a a fine word, a grand word for this situation.

$\mathrm{S} 3 \mathrm{~b}$ 形容目前的情况再贴切不过了。

Space here means the screen size, the words amount and the number of the lines listed in the subtitle. Generally, there should be a maximum of two lines for English and one line for Chinese at a time, in order to save the size of the screen space. And the Chinese characters for each line should be less than 13. Abundant loading of the words on the screen would affect the aesthetic art of the film or teleplay. Moreover, it is difficult for the audience to read the long subtitles on a screen in a limited time, as they are enjoying the moving images. That's why the subtitling must be simple, clear, adequate and concise.

Time restriction has mainly two implications, one is the duration of the sounds or images and the other one is the average time needed by the viewer for browsing through the whole lines. And the time spent on the subtitles is different because of the different backgrounds of the viewers. Some are not well educated, so they will need more time on the understanding of the lines. So the reading time of the subtitle would be different. But according to the research from The Encyclopedia of Language and Linguistics, the time of a single line appeared on the screen should be as little as two seconds, and the two-line subtitle should be less than seven seconds (Asher \& Simpson, 1994). Therefore, the average time of browsing subtitles should also be controlled. Such meaningless words are usually omitted during the translation. In some cases, the lack of lasting time or screen space has made the omission not a need but a requirement. Yet this kind of omission normally wouldn't hurt the audience's comprehension a bit. According to Relevance Theory, during this kind of communication (contents presented through big screen and the audience), language is not the sole carrier of purpose, other materials like sound, context, visual signal would complement the messages that are unable to be fully conveyed through language, the audience would process the information under the pattern of the ostensive-inferential communication, by combining all usable information and inferring the implicature on their own. In s1a, the original sentence stress the time. The direct omission of' far away" doesn't affect the meaning expressed, while it can make the Chinese subtitle much simpler and shorter. The phrase "once upon a time" and "far far away" in this example were condensed into one Chinese word“很久以 前".It is not always necessary to do the literal translation because the audience can grasp the meaning. Such omission makes the target text much shorter and briefer, conveying the same information as the original text at the same time.

When we talk about relevance in subtitle translation, we mean to focus on the connection between the subtitles and viewers' cognitive context. The viewers watch the subtitles for the viewers' cognitive context. The viewers watch the 
subtitles for the visual information and their subtitle watching helps activate their already existed information which is relevant to the widely and popularly used in the subtitles. Since they are usually short and not difficult to understand, the target language viewers do not have to spend much time and effort on the analysis of the intended implied meaning of the original.

\subsubsection{Amplification}

We can read and appreciate a literature work many times but for film subtitles on the screen are fleeting. Therefore, for a subtitle translation activity translator should take the difference of language and culture into consideration and also pay attention to the constraints of time and space. Strategies of addition and omission are always applied in translation. Addition is add some sentences or world in order to express original text more exactly while omission is to delete redundant contents to make sentence understood easier. During the inter-lingual communication, there are usually lots of distinctions between the two sides of communicator. Different cultural background, various perspectives over one same object and the ability of comprehension have made the understanding sometimes very tricky like there are some places missing. Amplification is the approach trying to fill in the missing parts in the gap, the subtitle translator can achieve relevance and therefore make fluent communication by adding the "extra but necessary" information. E.g.

S4a You know we have a spare.

$\mathrm{S} 4 \mathrm{~b}$ 我们有一把备用钥匙。

S5a this one will be easy.

S5b 这个会很容易解决。

S6a Brighton, a word, please.

S6b 布莱顿, 近一步说话。

S7a The king raised the little girl by himself, grooming her to one day lead.

S7b 国王一个人抚养公主长大,培养她将来能够继承王位。

S8a No matter how many times I do it, I still get excited on my wedding day.

$\mathrm{S} 8 \mathrm{~b}$ 无论我结多少婚, 在婚礼当天我总是异常兴奋。

S9a What is it with this kingdom?

$\mathrm{S} 9 \mathrm{~b}$ 我们的王国到底怎么了?

Though the subtitles translation is mainly guided by the "economic principle" to reduce the amount of information that the target audience has to deal with, due to the language and culture differences, sometimes the appropriate addition is necessary. In S4a, from the contextual effect, we can know that spare means 备用钥匙。When the monster came to kill snow white, she didn't want to let their friend into trouble, so she knocked the door and fight against monster for herself. They want to help her, but they can't go out, one of dwarfs said this line, so translator uses additional strategy to translate it. As long as the translator does not violate the time and space constraints of the subtitles, choosing the addition strategy can make the hidden information explicit, so that the target language audience can achieve the optimal relevance.

\subsubsection{Domestication}

Nowadays, in translation fields there exist two main trends combating with each other: domestication and foreignization.

Domestication strategy refers to adopt transparent and fluent style to reduce the strangeness of the foreign text maximally for the target language readers. In the movie subtitles translation, it means that the translator should consider the target language culture as a destination, close to the target language audience, select the subtitles that 
cater for the target language culture to convey the content of the original, eliminate the source language cultural factors, highlight the cultural values of the target language, which can make the audience feel he is watching the original when watching the target language text. The subtitler should take the target language viewers' cognitive environment to make them with least processing cost grasp the intended meaning of the speakers' utterance as quickly as possible. E.g.

S10a There is none greater than the power to join two people together in matrimony.

$\mathrm{S} 10 \mathrm{~b}$ 最伟大的权利莫过于让相爱的人喜结连理。

The Phrase "join two people together" is an English expression. The subtitle translator tries to find its equivalence in Chinese. The relationship between two people has been familiar to Chinese viewers and vividly expresses the meaning. By using a term known to Chinese, the subtitle translator enriches the meaning and conveys the original intention and meaning to the target language viewers. We also could use word for word to translate it. It can be translate into: 没有比让两个人一起结婚生活这个更大的权利了。 However, if we translate in this way, the target language would seems to be so pale and boring. Compared with above two translations, it is absolutely better to choose former one, domestication, to translation this line. Because, domestication would be better to catch target audience's eyes and competitiveness of Mirror Mirror.

S11a You never stopped believing in yourself and grew into the woman knew you would always become.

$\mathrm{S} 11 \mathrm{~b}$ 你从未放弃相信自己, 我望女成风的期盼也终成现实。

The term "grew into woman" means to become more elegant, intelligent and smart. In this situation, this phrase doesn't suitable to literal translation. We also could use word for word to translate it. It can be translate into: 你从未 放弃相信自己, 希望她成为女人的梦想终将实现。However, if we translate in this way, the target language would seems to be ambiguous. In my point of view, this sentence wants to express the father's wish for daughter to have a good life. Therefore, adopting familiar expression is relevance enough for target language viewers to understand the utterances and communicative effect has been thus achieved under this context. They do not need to emphasize the foreign aspect of the original work provide a target language version to fill gaps in knowledge and make the narrative easy and accessible for the target audience. The film should be taken into consideration. The audience expressing habit and appreciation, too obscure to understand unfamiliar words and expressions, that the audience will lose interest, due to the cultural differences and translation of time and space limit, subtitle translation cannot be add, so in order to raise ticket real income, in the translation of films of foreignizing translation method is very necessary and very common method of translation, domesticating strategy can be closer to audience and film distance, be just perfect. Domestication can make a lively and succinct, cordial and lively, easy to the audience understand and accept.

S12a we are gonna sweep this kid right off his feet.

$\mathrm{S} 12 \mathrm{~b}$ 我们要让那个孩子拜倒在我的石榴裙下。

S13a I'm not a leader.

$\mathrm{S} 13 \mathrm{~b}$ 我不是当领导的料.

Translation“leader"into “当领导的料”in this context achieves a special humorous effect. Translation"sweep this kid right off his feet”into“拜倒在我的石榴裙下”。In this film, the queen love the prince, she wants to do whatever it takes to let him fall in love with herself. "Sweep this kid right off his feet" an English expression. The subtitle translator tries to find its equivalence in Chinese. This translated subtitle is relevant enough with the expected optimal relevance and the target language viewers spare less effort to understand.

\section{CONCLUSION}

In this paper, relevance theory is applied as the theoretical guidance and abundant authentic subtitles from Mirror Mirror are collected as examples to illustrate how and why the employment of techniques dealing with linguistic 
and cultural factors can be influenced by its relevance. According to the relevance theory the purpose of subtitle translation is to help the audience understand the content of the movie easily. Consequently, it is important for translators to adopt suitable principles and strategies in the process of translation.

The contributions of this paper are as follows: Firstly, different from other kinds of translation, subtitling is restricted by time, space and other techniques. Therefore when subtitling, subtitlers should pay more attention to these constraints. A careful study of these constraints and the process of film subtitle-making are necessary for every translator working in this field. Secondly, this paper gives some useful translation strategies, namely addition, paraphrase, omission, literal translation and so on. Therefore, subtitle translator could translate film subtitle followed these strategies. Last but not the least; abundant examples demonstrate that Relevance theory is one of the most possible theories to film subtitling.

On the other hand, the limitations of this paper cannot be ignored Though the author has conducted a detailed analysis of subtitle translation, there are still some limitations and inadequacies which will need a further improvement .Briefly, the paper only focuses on the English subtitle translation of only one film Mirror Mirror, whose analysis is far from adequacy. And the examples cited in this paper are chosen by the author herself, which may not be comprehensive to some degree.

Finally is the suggestions and further study of the paper. This paper is only a case study and no specific problems of film subtitle translation are solved in it. Accordingly, more works concerning this field, especially empirical studies for the reception of film subtitle translation, would be popular The focus for future research in this field may become the researches on variety of film translation, that is to say, to study all the elements as a whole including visual image, sound and colors, and the research will help to develop awareness and analysis of the integration of semiotic resources in audiovisual.

\section{BIBLIOGRAPHY}

[1] Asher, R. E. \& Simpson, J. M. (1994). The Encyclopedia of Language and Linguistics Volume 2. Pergamon Press.

[2] Ma Yujie. Study on the Subtitle Translation Strategies of The Wedding Banquet from the Perspective of Skopos Theory [D].Suzhou: Soochow University, 2010, 17.

[3] Baker, M. (1998). Routledge Encyclopedia of Translation Studies. London: Routledge.

[4] Guardini, P. (1998). Decision-making in Subtitling. Perspectives: Studies in Translatology, 1, 98.

[5] Sperber, D. \& Wilson, D. (1986 \&1995). Relavance: Communication and Cognition. Blackwell, Oxford and Harvard University Press.

[6] Wang Peng. A Brief Study on Domestication and Foreignization in Movie Subtitle Translation-Take Shawshank Redemption for Example, Jin Tian 2013(1).

[7] Wang Yuandan. Cultural View on Subtitle Translation of Hong Kong Films-Through A Chinese Ghost Story. [D]. Wu Han University of Technology, 2012, 10.

[8] Yang Wenting. On Subtitle Translation of Sitcoms ----A Case Study of The Big Bang Theory[J].OVERSEAS ENGLISH 2013(4)

[9] You Zedong. On Subtitle Translation of Internet Movies, [J]. NEW COUNTRYSIDE, 2011(4).

[10] Zhou Qingdi. Subtitle Translation with a Case Study of Scent of a Woman [J].THE YOUTH WRITERS, 2011(7). 